\title{
Inflammatory, anti-inflammatory and regulatory cytokines in relatively healthy lung tissue as an essential part of the local immune system
}

\author{
Zane Vitenberga, Mara Pilmane
}

\begin{abstract}
Background. The innate and adaptive immune systems in lungs are maintained not only by immune cells but also by non-immune tissue structures, locally providing wide intercellular communication networks and regulating the local tissue immune response.

Aims. The aim of this study was to determine the appearance and distribution of inflammatory, anti-inflammatory and regulatory cytokines in relatively healthy lung tissue samples.

Material and Methods. We evaluated lung tissue specimens obtained from 49 patients aged 9-95 years in relatively healthy study subjects. Tissue samples were examined by hematoxylin and eosin staining. Interleukin-1 (IL-1), interleukin-4 (IL-4), interleukin-6 (IL-6), interleukin-7 (IL-7), and interleukin-10 (IL-10) were detected by an immunohistochemistry (IMH) method. The number of positive structures was counted semiquantitatively by microscopy. Non-parametric tests were used to analyse the data.

Results. IL-1-positive cells were mostly found in the bronchial cartilage and alveolar epithelium. Immunoreactive lung macrophages were also found. The numbers of IL-4, IL-6, IL-7, and IL-10 containing cells were also found in the bronchial epithelium (in addition to those previously listed). The number of positive structures varied from occasional to moderate, but was graded higher in cartilage. Overall, fewer IL-1-positive cells and more IL-10-positive cells were found. Almost no positive structures for all examined cytokines were found in connective tissue and bronchial glands. Conclusions. Relatively healthy lung tissue exhibits anti-inflammatory response patterns. The cytokine distribution and appearance suggest persistent stimulation of cytokine expression in lung tissue and indicate the presence of local regulatory and modulating patterns. The pronounced cytokine distribution in bronchial cartilage suggests the involvement of a compensatory local immune response in the supporting tissue.
\end{abstract}

Key words: cytokines, lung, immunity, immunohistochemistry

Received: December 30, 2016; Accepted with revision: May 26, 2017; Available online: June 5, 2017

https://doi.org/10.5507/bp.2017.029

Department of Morphology, Institute of Anatomy and Anthropology, Riga Stradins University, Kronvalda Boulevard 9, Riga, LV-1010, Latvia Corresponding author:Zane Vitenberga, e-mail:Zane.Vitenberga@rsu.Iv

\section{INTRODUCTION}

The complexity of the lung immune system extends beyond immunocompetent cells and their wide intercellular signalling networks. Lung tissue immunity harbours necessary structures and forces for pathogen recognition, also maintaining various immune response types and tissue repair. In general, the lung innate and adaptive immune system in its steady state exhibits a generally anti-inflammatory environment ${ }^{1}$. Failure of immune response regulation might lead to a disrupted normal lung tissue architecture and structure due to minor and major structural changes ${ }^{2}$.

The barrier functions of epithelial tissue in the lung environment are essentially responsible for a major portion of tissue homeostasis ${ }^{3}$. Epithelial tissue works as an outline barrier in the respiratory system ${ }^{3,4}$. Apart from true immune cells, epithelial tissue and its communication with other tissue structures design and shape signalling pathways to produce local immunity ${ }^{5}$. We can highlight the novel understanding of immune system, for which immunogenic properties have been described in other than immunocompetent cells. Interestingly, epithelial tissue is among the structures that possess immunogenicity ${ }^{6,7}$.

The airway epithelium works as a physical barrier in lungs to maintain effective mucociliary clearance ${ }^{8,9}$. Mucus covers the upper surface of the epithelium and actively serves as an environment capable of preventing airways from possible inhaled harm ${ }^{8,10}$.

Macrophages resident in non-immune tissue participate in tissue-specific homeostasis. Interestingly, tissuespecific macrophages able to secrete extensive types of various biological molecules provide unique landscapes both in steady and active states, mirroring the ontogenetic and microenvironment background related to their role in shaping local homeostasis ${ }^{11,12}$. Moreover, tissue macrophages residing in certain organ-specific environments display high plasticity and may shape their cellular identity ${ }^{13}$.

The interleukin-1 (IL-1) family has been found to have auto-inflammatory and autoimmune properties; however, IL-1 itself is a pro-inflammatory cytokine. IL-1 regulates the innate and adaptive immune system towards the initiation and amplification of the immune response into inflammation and regulates tissue damage. As a result 
of the IL-1 presence in tissue, the recruitment of macrophages is a local immune response ${ }^{14}$.

IL-4 is a key cytokine in the development of allergic inflammation and an important local factor participating in cell signalling due to allergen exposure ${ }^{15}$. IL-4 increases fibroblast proliferation and the secretion of extracellular matrix components leading to fibrosis ${ }^{16}$. IL-4 also highly promotes the pathogenesis of both allergy and asthma ${ }^{17}$.

IL-6 is a pro-inflammatory cytokine; however, the important anti-inflammatory and regenerative nature of IL-6 has also been described ${ }^{18}$. Furthermore, the IL-6-mediated profibrotic properties through the direct activation of fibroblasts and numerous indirect mechanisms have been reviewed ${ }^{16,19}$.

IL-7 is also a cytokine with pleiotropic functions; however, IL-7 mostly functions as an immunoregulatory cytokine. Wide research has established IL-7 as the regulator of lymphocyte overall development, maintenance and homeostasis ${ }^{20}$. IL-7 increases lymphoid cell lineage re-population and generation, serving as a lymphoid regenerative factor ${ }^{21}$. Cytokine IL-7 is involved in the early development and further maintenance of $\mathrm{T}$ cells ${ }^{17,21}$.

Immunity concepts have been reviewed with interest when considering the possible non-immune cell response to various inflammatory mediators as well as the production of their own pro-inflammatory, regulatory and antiinflammatory mediators, not only by macrophages and in surface structures but also deeper in the tissue underlying the epithelium.

Thus, the aim of the present study was to perform a routine histological analysis and determine the appearance and relative distribution of interleukins (IL)-1, IL-4, IL-6, IL-7, and IL-10 in relatively normal lung tissue material.

\section{MATERIALS AND METHODS}

\section{Patients}

We evaluated lung tissue material obtained during a post mortem autopsy from forty-nine patients aged 9 to 95 years in relatively healthy study subjects. Among the diagnoses of the study subjects were heart disease (mostly sudden cardiac death) or death of the patients by either intentional self-harm (suicide) or unintentional major injury due to trauma. Tissues with surgically and histologically clear resection margins of suspected cancer patients were included in this study. Patients with severe respiratory conditions and failure, with any history of acute or severe chronic respiratory pathology, and treated with medications conflicting the possible results of this study, as well as tissue samples with pathological findings determined in routine histological analysis, were not included in this study.

All authors hereby declare that all experiments were examined and approved by the appropriate ethics committee and were therefore performed in accordance with the ethical standards laid down in the 1964 Declaration of Helsinki. This study was approved by the Ethical Committee of Pauls Stradins Clinical University Hospital dated January 23, 2013.

\section{Methodology}

Soft tissue material pieces with an approximate size of $1 \mathrm{~cm}^{3}$ were taken during the autopsy and further fixed in a mixture of $2 \%$ formaldehyde and $0.2 \%$ picric acid in $0.1 \mathrm{M}$ phosphate buffer ( $\mathrm{pH}$ 7.2). Afterwards, the tissue samples were rinsed in thyroid solution, containing $10 \%$ sucrose for $12 \mathrm{~h}$. Continuing the rinsing, tissue specimens were embedded in paraffin. Six to seven micrometre $(\mu \mathrm{m})$ thin tissue sections were cut to proceed with staining. Routine histological staining with hematoxylin and eosin was used for each case to obtain review photographs of each tissue material. All the tissue specimens were examined by bright field microscopy.

From sixty-nine tissue materials in total, we excluded twenty tissue specimens because pathological findings (e.g., massive immune cell infiltrations, bleeding, emphysema) were observed by bright field microscopy, despite the clear medical history.

Forty-nine tissue sections were prepared for the detection of cytokines IL-1 $\alpha$, IL-4, IL-6, IL-7, and IL-10 using the biotin-streptavidin immunohistochemistry (IMH) method $^{22}$. The characteristics and parameters of the primary antibodies used in this study were as follows:

- IL-1 $\alpha$ (code: sc-9983, mouse, work dilution 1:50, Santa Cruz Biotechnology),

- IL-4 (code: orb10908, rabbit, work dilution 1:100, Biorbyt),

- IL-6 (code: sc-130326, mouse, work dilution 1:50, Santa Cruz Biotechnology),

- IL-7 (code: orb48420, rabbit, work dilution 1:100, Biorbyt),

- IL-10 (code: P22301, rabbit, work dilution 1:400, BioSite).

The samples were examined under bright field microscopy using a Leica DC 300F camera microscope for conventional histological photographs. The acquired photographs were analysed using the picture visualization program Image Pro Plus.

We examined the appearance and distribution of cytokine-containing structures, where the relative number of positive immunohistochemical structures in the epithelial tissue (precisely epithelial cells, both ciliated and secretory), connective tissue (fibroblasts), bronchial glands (both serous and mucous glandulocytes), bronchial cartilage (chondroblasts, chondrocytes), and alveolar epithelium, as well as cytokine-containing alveolar macrophages, were graded semi-quantitatively ${ }^{23,24}$.

The following scale of the semi-quantitative method was used, counting the immunoreactive (positive) structures seen in the visual field: 0 - no positive structures (0\%), $0 /+-$ occasional positive structures $(12.5 \%),+-$ few positive structures $(25 \%),+/++-$ few to a moderate number of positive structures $(37.5 \%),++-$ moderate number of positive structures $(50 \%),++/+++-$ moderate number of to numerous positive structures $(62.5 \%),+++$ - numerous positive structures $(75 \%),+++/++++-$ numerous to abundant positive structures $(87.5 \%)$, ++++ - abundance of positive structures (100\%) observed in the visual field. 


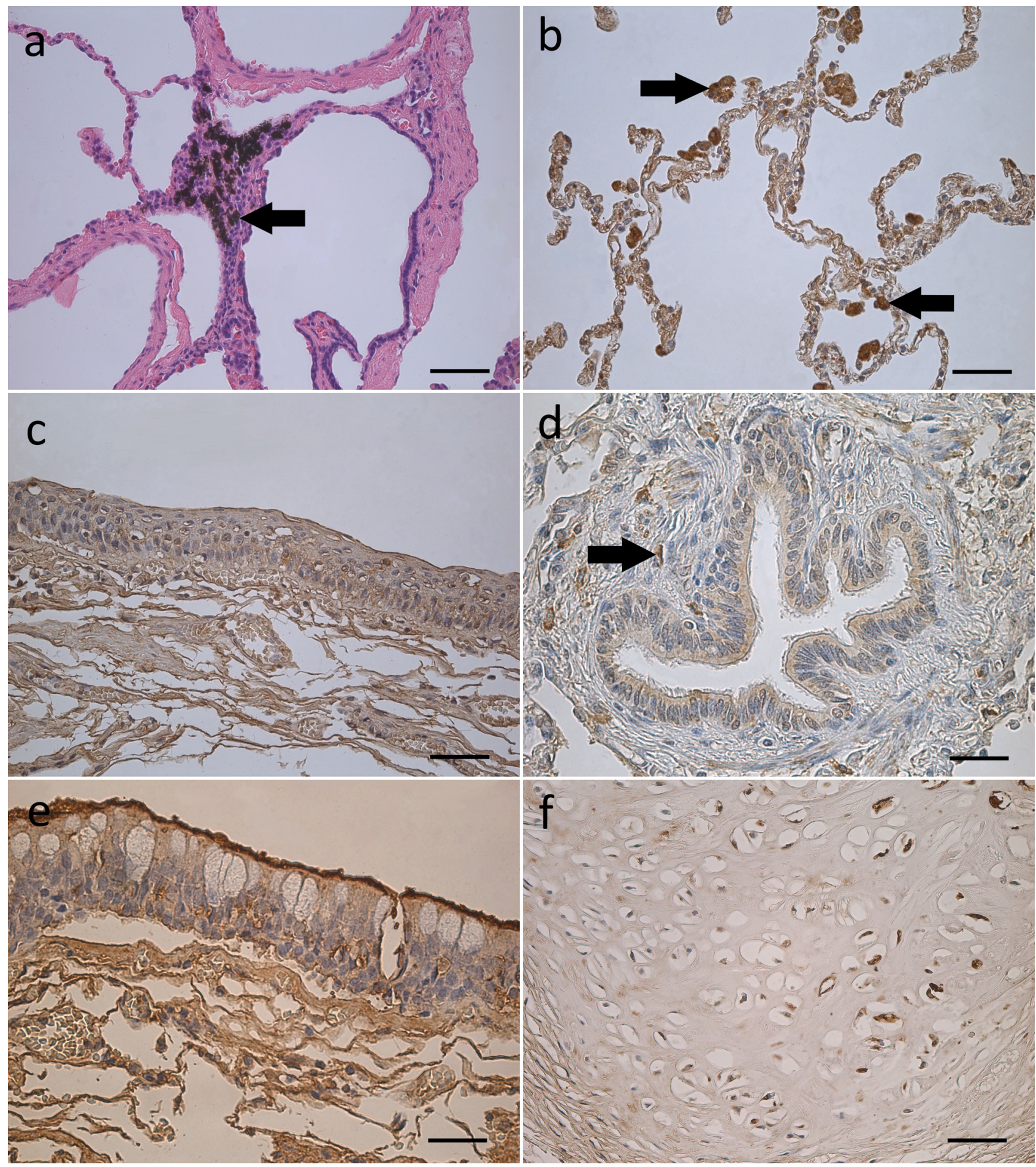

Fig. 1. Representative microphotographs of relatively healthy lung tissue examined by routine histological staining with hematoxylin and eosin (a) and detection of cytokines IL-1 $\alpha$, IL-4, IL-6, IL-7, and IL-10 using the biotin-streptavidin immunohistochemistry (IMH) method (b - f). (a) An arrow marks dust-containing macrophages in the lung tissue of a 29-year-old male. Hematoxylin and eosin, X250. Scale bar, $16 \mu \mathrm{m}$. (b) Note the numerous IL-4-containing alveolar epithelial cells and alveolar macrophages (arrows) in the lung tissue of a 56-year-old male. IL-4 IMH, X200. Scale bar, $20 \mu \mathrm{m}$. (c) Note the moderate number of IL-6-containing bronchial epithelial cells in the squamous metaplastic epithelium in the lung tissue of a 45-year-old male. IL-6 IMH, X250. Scale bar, $16 \mu \mathrm{m}$. (d) Note the moderate IL-7-containing bronchial epithelial cells and few connective tissue fibroblasts indicated with an arrow (d) in the lung tissue of an 81-year-old female. IL-7 IMH, X400. Scale bar, $10 \mu \mathrm{m}$. (e) Note the moderate IL-10-containing bronchial epithelial cells in the lung tissue of a 27-year-old female. IL-10 IMH, X400. Scale bar, $10 \mu \mathrm{m}$. (f) Note the moderate IL10 -containing hypertrophic hyaline cartilage cells in a large bronchus wall of a 30-year-old male. IL-10 IMH, X250. Scale bar, $16 \mu$ m. 
Table 1. Appearance and distribution of cytokines in 49 lung tissue specimens of relatively healthy humans.

\begin{tabular}{|c|c|c|c|c|c|c|c|c|c|c|c|c|c|c|c|c|c|c|}
\hline & \multicolumn{3}{|c|}{$\begin{array}{l}\text { Bronchial } \\
\text { epithelium }\end{array}$} & \multicolumn{3}{|c|}{$\begin{array}{l}\text { Connective } \\
\text { tissue }\end{array}$} & \multicolumn{3}{|c|}{$\begin{array}{l}\text { Bronchial } \\
\text { glands }\end{array}$} & \multicolumn{3}{|c|}{$\begin{array}{l}\text { Bronchial } \\
\text { cartilage }\end{array}$} & \multicolumn{3}{|c|}{$\begin{array}{l}\text { Alveolar } \\
\text { epithelium }\end{array}$} & \multicolumn{3}{|c|}{$\begin{array}{c}\text { Alveolar } \\
\text { macrophages }\end{array}$} \\
\hline & Mdn & $\mathrm{Q}_{1}$ & $\mathrm{Q}_{3}$ & Mdn & $\mathrm{Q}_{1}$ & $\mathrm{Q}_{3}$ & Mdn & $\mathrm{Q}_{1}$ & $\mathrm{Q}_{3}$ & Mdn & $\mathrm{Q}_{1}$ & $\mathrm{Q}_{3}$ & Mdn & $\mathrm{Q}_{1}$ & $\mathrm{Q}_{3}$ & Mdn & $\mathrm{Q}_{1}$ & $\mathrm{Q}_{3}$ \\
\hline IL-1 & 0.5 & 0 & 1.0 & 0.5 & 0 & 1.0 & 0 & 0 & 0 & 1.0 & 0.5 & 1.0 & 0.5 & 0.5 & 1.5 & 0.5 & 0.5 & 1.0 \\
\hline IL-4 & 1.5 & 0.75 & 1.5 & 0.5 & 0 & 0.5 & 0.5 & 0 & 1.0 & 0.5 & 0.5 & 1.0 & 1.0 & 0.5 & 1.75 & 1.5 & 0.5 & 2.0 \\
\hline IL-6 & 1.0 & 1.0 & 2.5 & 0.5 & 0 & 0.5 & 0 & 0 & 0 & 1.0 & 0.5 & 2.0 & 1.0 & 0.5 & 1.0 & 1.5 & 0.5 & 2.0 \\
\hline IL-7 & 1.25 & 1.0 & 1.5 & 0.5 & 0 & 1.0 & 0.5 & 0 & 0.5 & 2.0 & 1.5 & 2.5 & 1.0 & 0.75 & 2.0 & 0.75 & 0.5 & 2.0 \\
\hline IL-10 & 1.25 & 1.0 & 1.5 & 1.0 & 0.5 & 1.0 & 0.5 & 0 & 1.0 & 1.75 & 1.5 & 2.5 & 1.0 & 0.5 & 2.0 & 1.5 & 1.0 & 2.0 \\
\hline
\end{tabular}

“0" - no positive $(0)$ structures seen in the visual field, “ 0.5 " - rare $(0 /+)$ positive structures seen in the visual field, " 1.0 " - few $(+)$ positive structures seen in the visual field, " 1.5 " - few to a moderate $(+/++)$ number of positive structures seen in the visual field, " 2.0 " - moderate (++) number of positive structures seen in the visual field, " 2.5 " - moderate to numerous $(++/+++)$ positive structures seen in the visual field, " 3.0 " - numerous $(+++)$ positive structures seen in the visual field, "IL-1" - interleukin-1, "IL-4" - interleukin-4, "IL-6" - interleukin-6, "IL-7" - interleukin-7, and “IL-10" - interleukin-10, “Mdn” - Median value, “Q” ${ }_{1}-25^{\text {th }}$ percentile value (1 ${ }^{\text {st }}$ Quartile), “Q”" $-75^{\text {th }}$ percentile value $\left(3^{\text {rd }}\right.$ Quartile).

All the acquired data were ranking as ordinal values, where no positive structures $(0)$ seen in visual field of bright field microscopy were ranked with the value of 0 , occasional positive structures $(0 /+)$ were ranked with the value of 0.5 , few positive structures $(+)$ were ranked with the value 1.0, et cetera. The maximum grade of abundance of positive structures (++++) in the visual field was ranked with the value of 4.0 .

To perform the statistical analysis, we used nonparametric statistical methods.

- From the parameters analysed in descriptive statistics, the median (Mdn) value and interquartile range (IQR) were determined.

- The Wilcoxon matched pairs Signed Rank Test was conducted to determine whether there was a difference in the number of positive structures of one examined cytokine (dependent variable) in two different tissue types (independent variables, e.g., related groups), as well as to evaluate the positive ranks, negative ranks and ties. The data are medians unless otherwise stated.

- Spearman's rank-order correlation was run to determine the relationship between the number of cytokine-containing structures in one type of tissue and in a different tissue, as well as with age. We evaluated the correlation coefficient as follows: The closer $r_{s}$ (Spearman's rho) is to +1 , the stronger the positive correlation. The closer $r_{s}$ is to -1 , the stronger the negative correlation. The $r_{s}$ value of $0.00-0.30$ is regarded as a negligible correlation; $0.30-0.50$, as low; $0.50-0.70$, as moderate; $0.70-0.90$, as strong; and $0.90-1.00$, as a very high (very strong) correlation ${ }^{25}$.

The statistical analysis was performed using the statistical program SPSS Statistics, version 22.0 (IBM Company, Chicago, USA). In all the statistical analyses, $P$ values $<0.05$ were considered statistically significant.

\section{RESULTS}

\section{Routine histology}

Among the minor pathological findings, we evalu- ated emphysematous lung tissue regions not larger than one visual field of bright field microscopy in two elderly patients. In the tissue materials of three patients, increased smooth muscle cell proliferation was seen as a minor pathological finding in the wall of a conducting bronchus or bronchiole. Neoangiogenesis with few smallcalibre capillaries in the lung tissue parenchyma was seen in one case. Sclerotized blood vessels were found in the tissue materials of three elderly patients; however, these findings were rare in the whole tissue material. We found hemosiderin-containing macrophages and dust-containing macrophages (Fig. 1a) in three patients.

Interestingly, we found goblet cell hyperplasia in the tissue material of one patient as a fragment of the lining epithelium with a size not exceeding one visual field at magnification X200. Goblet cell hyperplasia was seen as a unique lining epithelium covering the wall of a largecalibre bronchus. Normal ciliated cells were partially replaced by an abundance of goblet cells.

\section{Immunohistochemistry}

The number of IL-1-positive cells in the bronchial epithelium and connective tissue varied from no positive structures $(0 \%)$ seen in the visual field to few-to-moderate $(+/++$ or $37.5 \%)$ IL-1-containing structures (Fig. 1b). Almost no positive (0\%) structures for IL-1 were detected in bronchial glands. Surprisingly, IL-1-containing structures in the bronchial cartilage were found more frequently. The number of IL-1-positive alveolar epithelial cells varied from no positive structures in the visual field to a moderate number (++ or $50 \%$ ) observed. The count of IL-1 immunoreactive macrophages was also found to be variable, numbering from occasional $(0 /+$ or $12.5 \%)$ to almost numerous (+++ or 75\%) IL-1-containing macrophages. In summary, more IL-1 positive tissue structures were found in bronchial cartilage and alveolar epithelium, ranging from occasional $(0 /+$ or $12.5 \%)$ to few (+ or $25 \%)$ immunoreactive structures seen in the visual field. No positive $(0 \%)$ to only a few (+ or $25 \%)$ positive cells were found in the bronchial epithelium, connective tissue and bronchial glands.

IL-4-containing bronchial epithelial cells varied from 
Table 2. Summary of Spearman's rank-order correlation analysis to determine the strong relationship $(0.70>\mid$ rs $\mid>0.90)$ between the numbers of cytokine-containing structures in different tissues. The results are ordered in descending order by correlation coefficient rs (Spearman's rho).

\begin{tabular}{llccc}
\hline \multicolumn{2}{l}{ Tissue group (evaluated cytokine) } & $\mathrm{n}$ & $\mathrm{r}_{\mathrm{s}}$ & $P$ \\
\hline Alveolar epithelium (IL-1) & Alveolar epithelium (IL-4) & 24 & 0.823 & $<0.0005$ \\
Alveolar epithelium (IL-1) & Bronchial glands (IL-6) & 18 & 0.809 & $<0.0005$ \\
Alveolar epithelium (IL-4) & Alveolar epithelium (IL-10) & 24 & 0.804 & $<0.0005$ \\
Alveolar epithelium (IL-1) & Alveolar epithelium (IL-10) & 24 & 0.778 & $<0.0005$ \\
Bronchial epithelium (IL-10) & Alveolar epithelium (IL-10) & 19 & 0.769 & $<0.0005$ \\
Alveolar epithelium (IL-1) & Alveolar epithelium (IL-7) & 24 & 0.767 & $<0.0005$ \\
Alveolar epithelium (IL-6) & Bronchial glands (IL-10) & 18 & 0.763 & $<0.0005$ \\
Alveolar macrophages (IL-4) & Bronchial glands (IL-10) & 19 & 0.742 & $<0.0005$ \\
Alveolar epithelium (IL-1) & Bronchial epithelium (IL-10) & 19 & 0.715 & 0.001 \\
Alveolar epithelium (IL-1) & Bronchial glands (IL-10) & 18 & 0.711 & 0.001 \\
\hline
\end{tabular}

“n” - pairs analysed in Spearman's rank-order correlation analysis, rs - correlation coefficient (Spearman's rho), " $P$ ” - $P$ values $<0.05$ are considered statistically significant, "IL-1" - interleukin-1, "IL-4" - interleukin-4, "IL-6” - interleukin-6, "IL-7” - interleukin-7, and "IL-10" - interleukin-10.

occasional ( $0 /+$ or $12.5 \%)$ to numerous (+++ or $75 \%)$ IL-4 positive cells. The number of IL-4-containing fibroblasts in the connective tissue varied from no positive $(0 \%)$ structures to a moderate number (++ or $50 \%)$, except for two cases, where we evaluated numerous $(+++$ or $75 \%)$ IL-4-positive fibroblasts. Almost no positive (0\%) to occasional $(0 /+$ or $12.5 \%)$ IL-4 positive glandulocytes and connective tissue fibroblasts were found in this study. Overall, the appearance of IL-4 positive structures in the bronchial cartilage and alveolar epithelium was graded from no positive $(0 \%)$ structures to a moderate number (++ or $50 \%)$. The number of IL-4-containing alveolar macrophages varied from occasional $(0 /+$ or $12.5 \%)$ to almost numerous (+++ or $75 \%)$.

The appearance and distribution of IL- 6 among the tissue groups of this study varied, ranging from occasional $(0 /+$ or $12.5 \%)$ structures in the connective tissue and in the bronchial glands and mostly few (+ or $25 \%$ ) structures in the alveolar epithelium. IL-6-containing structures were more prominent in the bronchial epithelium (Fig. 1c) and in the bronchial cartilage, mostly ranging from few to moderate $(+/++$ or $37.5 \%)$. In addition, few to moderate IL-6-containing macrophages were found in the majority of the examined tissue specimens.

Similar findings were seen in the analysis of the appearance and distribution of IL-7-positive structures (Fig. 1d), except for IL-7-containing alveolar epithelium, where few to moderate $(+/++$ or $37.5 \%)$ IL-7 positive epithelial cells were found. Interestingly, from a moderate (++ or $50 \%)$ to numerous (+++ or $75 \%$ ) IL-7 positive structures were found in bronchial cartilage, reaching overall the highest evaluation among all the examined cytokines.

The number of IL-10-containing structures in bronchial epithelium (Fig. 1e), in alveolar epithelium, as well as the number of alveolar macrophages varied from occasional $(0 /+$ or $12.5 \%)$ to numerous $(+++$ or $75 \%)$ structures seen in visual field, in average ranging from few to moderate $(+/++$ or $37.5 \%)$ number. Only occasional (0/+ or $12.5 \%)$ IL-10-containing glandulocytes were found in this study. Few (+ or 25\%) fibroblasts positive for IL10 were found in majority of examined tissue specimens, where this result was seen as the most prominent among all cytokines evaluated in connective tissue. Moderate (++ or $50 \%$ ) number of mostly IL-10-containing hypertrophic chondrocytes was found in bronchial cartilage (Fig. 1f).

Overall, the number of IL-4, IL-6, IL-7, IL-10 positive structures was considerably higher in the cartilage, alveolar epithelium, and bronchial epithelium; furthermore, there was a more pronounced number of lung macrophages. No positive to few structures were found in the connective tissue and bronchial glands (Table 1).

\section{Statistical data}

We found a statistically significant $(P<0.05)$ lower number of IL-1 positive structures in bronchial glands (0), connective tissue and bronchial epithelium (0.5). A higher number of IL-1-containing structures were found in the bronchial cartilage; in addition, more alveolar macrophages (1.0) were observed to containing IL-1. IL-1positive cells in the bronchial glands were fewer than in

$\mathrm{IL}-1 \alpha$

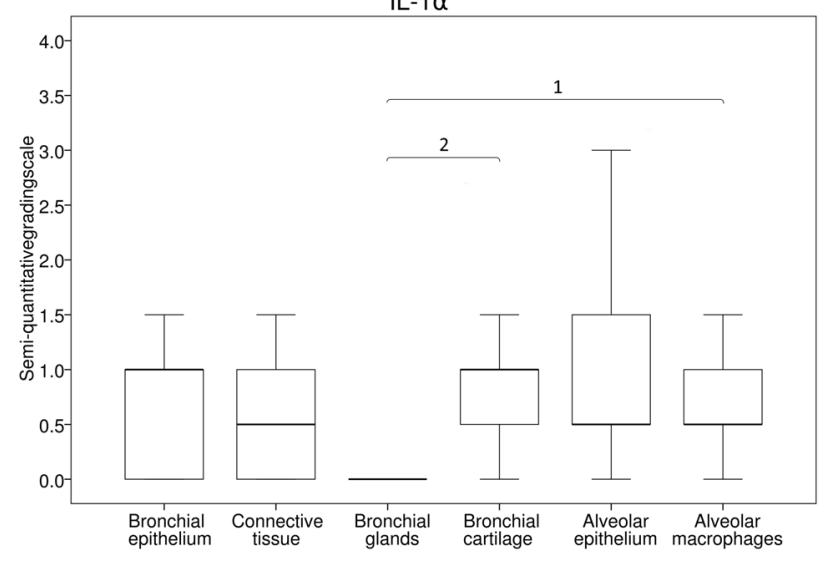

Fig. 2. Comparison of IL-1 $\alpha$-containing structures in bronchial glands, bronchial cartilage and of the number of IL- $1 \alpha$-positive alveolar macrophages. " 1 " $-\mathrm{z}=-3.596, P<0.0005, \mathrm{n}=18$; " 2 " $-\mathrm{z}=-4.433, P<0.0005, \mathrm{n}=38$. 


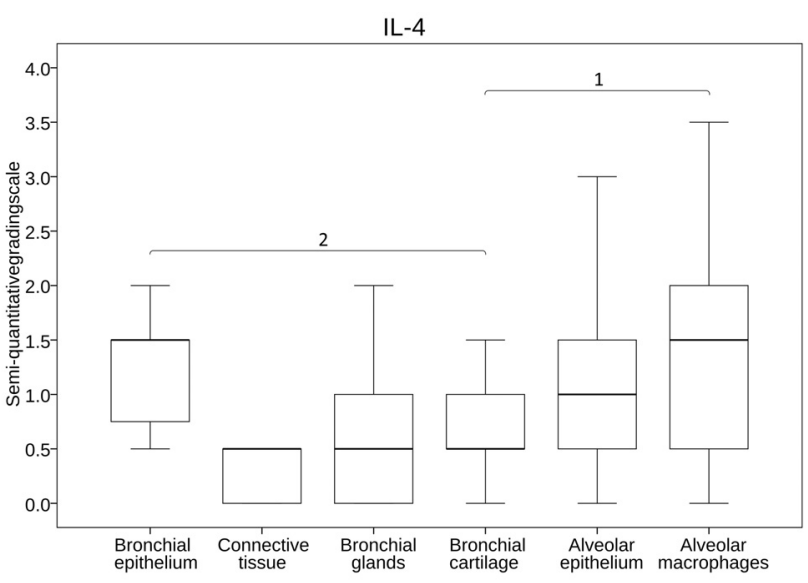

Fig. 3. Comparison of IL-4-containing structures in bronchial cartilage, bronchial epithelium and of the number of IL-4-containing alveolar macrophages. " 1 " $-\mathrm{z}=-2.263, P=0.024, \mathrm{n}=$ 20; “2”- $\mathrm{z}=-2.585, P=0.01, \mathrm{n}=32$.

IL-7

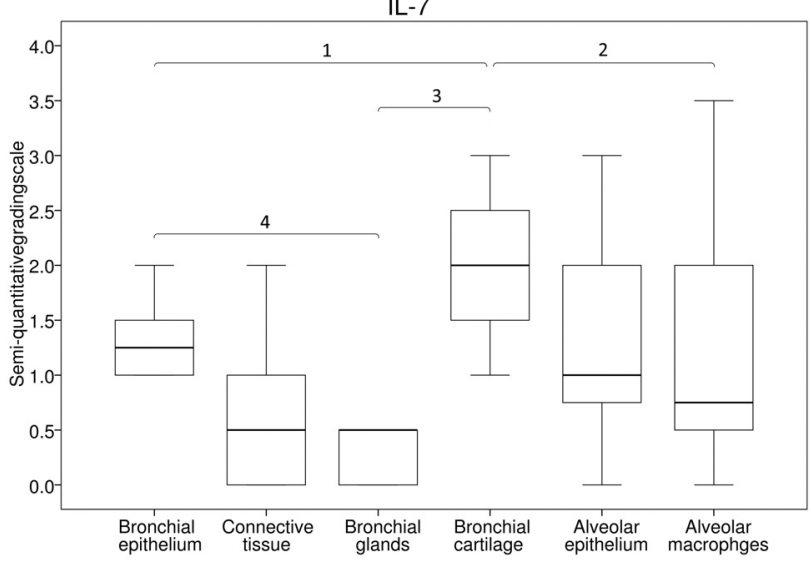

Fig. 5. Comparison of IL-7-containing structures in bronchial epithelium, bronchial glands, bronchial cartilage and of the number of IL-7-containing alveolar macrophages. " 1 " $-\mathrm{z}=$ -4.181, $P<0.0005, \mathrm{n}=31 ;$; 2 ” $-\mathrm{z}=-2.762, P=0.006, \mathrm{n}=20$; “3”- $\mathrm{z}=-5.260, P<0.0005, \mathrm{n}=39 ; “ 4$ ” $-\mathrm{z}=-3.595, P<0.0005$, $\mathrm{n}=30$.

the bronchial cartilage and were fewer compared with the number of alveolar macrophages (Fig. 2).

A statistically significant $(P<0.05)$ lower number of IL-4-containing structures were found in the connective tissue, bronchial glands and bronchial cartilage (0.5); however, a higher number of IL-4 positive structures were found in the alveolar epithelium (1.0) and bronchial epithelium, and there was a higher number of IL-4-containing macrophages (1.5). IL-4-positive cells in the bronchial cartilage were fewer than in the bronchial epithelium and were fewer compared with the number of alveolar macrophages (Fig. 3).

Significantly $(P<0.05)$ fewer IL-6-positive structures were found in the bronchial glands $(0)$ and connective tissue (0.5); however, more IL-6-positive structures were found in the bronchial epithelium, bronchial cartilage,

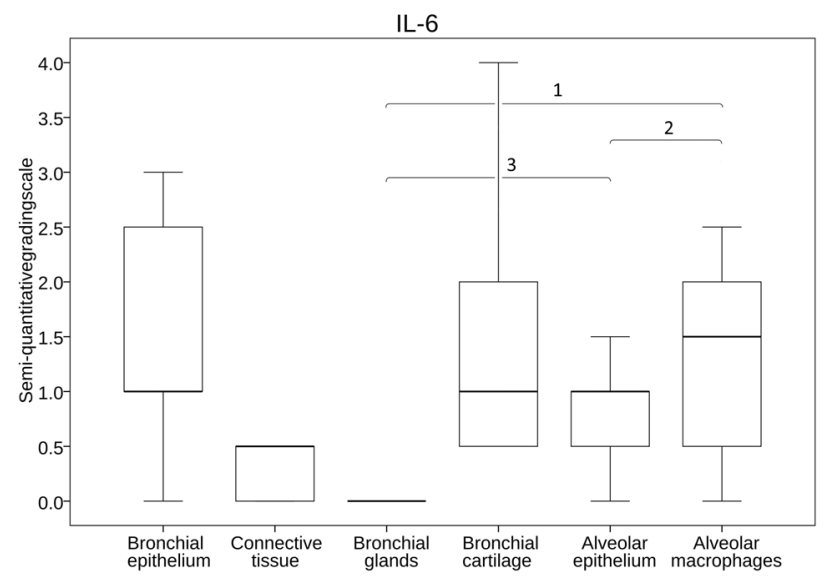

Fig. 4. Comparison of IL-6-containing structures in bronchial glands, alveolar epithelium and of the number of IL-6-containing alveolar macrophages. "1" $-\mathrm{z}=-3.539, P<0.0005, \mathrm{n}=18$; “2” $-\mathrm{z}=-2.275, P<0.023, \mathrm{n}=24$; “3” $-\mathrm{z}=-3.487, P<0.0005$, $\mathrm{n}=18$.

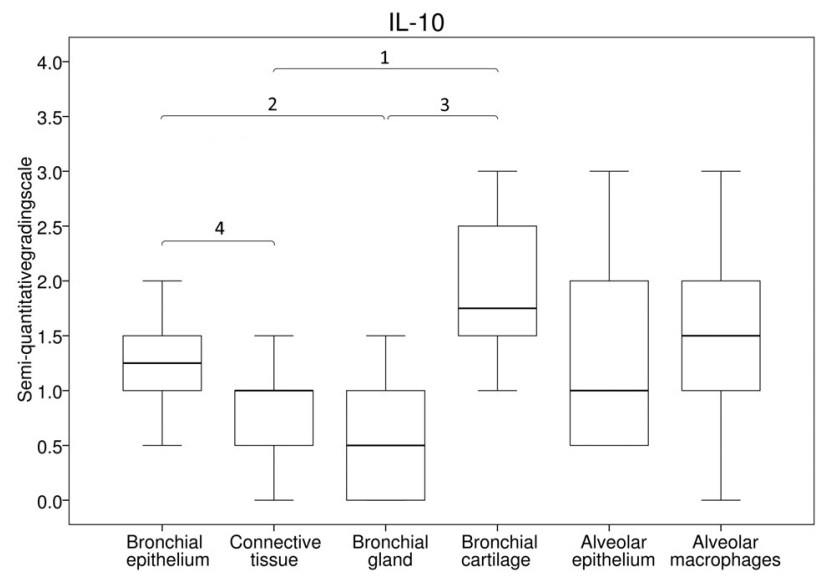

Fig. 6. Comparison of IL-10-containing structures in bronchial epithelium, connective tissue, bronchial glands and bronchial cartilage. " 1 " $-\mathrm{z}=-5.335, P<0.0005, \mathrm{n}=42$; " 2 " $-\mathrm{z}=-4.191$, $P<0.0005, \mathrm{n}=31$; “3” $-\mathrm{z}=-5.277, P<0.0005, \mathrm{n}=38$; “4” $-\mathrm{z}$ $=-4.081, P<0.0005, \mathrm{n}=37$.

and alveolar epithelium (1.0), as well as IL-6 containing alveolar macrophages (1.5). IL-6-positive cells in bronchial glands were fewer than in the alveolar epithelium and were fewer compared with the number of alveolar macrophages (Fig. 4).

A statistically significant $(P<0.05)$ lower number of IL-7-containing structures was found in the connective tissue, bronchial glands (0.5) and alveolar epithelium (1.0). A higher number of IL-7-containing structures were found in the bronchial epithelium (1.5) and in the bronchial cartilage (2.0), as well as a higher number of IL-7 positive alveolar macrophages (1.5). IL-7-positive cells in the bronchial glands were fewer than in the bronchial epithelium or bronchial cartilage and were fewer compared with the number of alveolar macrophages (Fig. 5).

Similarly, regarding the IL-7 distribution among the 
examined tissues, a statistically significant $(P<0.05)$ lower number of IL-10-containing structures were found in the bronchial glands $(0.5)$, connective tissue and alveolar epithelium (1.0). Furthermore, a higher number of IL-10-containing structures were found in the bronchial epithelium (1.5) and bronchial cartilage (2.0), as well as a higher number of IL-7-positive alveolar macrophages (1.5). IL-10-positive cells in the bronchial glands and connective tissue were fewer than those in the bronchial epithelium and bronchial cartilage (Fig. 6).

A statistically significant $(P<0.05)$ positive correlation with a strong relationship was found between the number of positive structures for mostly IL-1 and IL-10 in the alveolar epithelium, as well as between the number of positive structures for IL-10 in bronchial epithelium and bronchial glands with positive structures for IL-4, IL-6 and IL-7. Interestingly, there was a strong, positive correlation between positive structures for IL-1 and IL-10 in the alveolar epithelium $\left(\mathrm{r}_{\mathrm{s}}=0.778, P<0.001\right)$, alveolar epithelium and bronchial epithelium $\left(\mathrm{r}_{\mathrm{s}}=0.715, P=\right.$ 0.001 ), as well as alveolar epithelium and bronchial glands $\left(\mathrm{r}_{\mathrm{s}}=0.711, P=0.001\right)$, respectively (Table 2$)$.

No statistically significant $(P<0.05)$ positive or negative correlations were determined between the number of positive structures in any tissue groups examined in this study and age.

\section{DISCUSSION}

Our study reveals a more pronounced number of IL-1containing structures in bronchial cartilage, as well as a higher number of IL-1-containing alveolar macrophages; however, fewer IL-1-positive structures were found in the bronchial epithelium, connective tissue and in alveolar epithelium. We also measured a strong correlation of exclusively IL-1-containing alveolar epithelium dependent on other tissue groups containing predominantly IL-10, as well as IL-4, IL-6, and IL-7. Although we may speculate about the substantiation of IL-1 regulating all other cytokines, conversely, the release of IL-1 is also regulated by other pro-inflammatory, regulating and anti-inflammatory cytokines; therefore, the causality of one cytokine affecting another cytokine must be estimated in the context of multiple factors. In summary, epithelial cells are both activated by and further release the following cytokines: IL-1 $\alpha$, IL-4, IL-6 and IL-10 $0^{3,5-7,26}$. As the first exposed surface, the airway epithelium actively regulates local immunity. Due to antigen exposure, activated epithelial cells might recruit locally inhabiting immunocompetent cells. Epithelial cells use autocrine and paracrine signalling pathways to provide intercellular and intracellular communication $^{3,4}$. In summary, epithelial tissue serves as a first-line defence system in the innate immune system due to the continuous exposure to airborne antigens, sensing potential danger and harm, while initiating, regulating and suppressing the adaptive immunity ${ }^{3}$. Pro-inflammatory cytokines (IL-1, IL-6) and chemokines produced by epithelial cells recruit and activate immune cells in the submucosa $a^{5}$ Importantly, specifically IL- $1 \alpha$ even boosts the epithelial cell participation in the immune response through the autocrine stimulation ${ }^{8}$. However, to suppress the immune responses caused by the release of IL-1, the epithelium might release anti-inflammatory cytokine IL$10^{3}$. Therefore, we suggest that in relatively normal lung tissue, the anti-inflammatory environment is produced by the down-regulation of pro-inflammatory cytokines.

We found a higher number of IL-4-containing structures in the bronchial epithelium and alveolar epithelium but a slightly lower number of structures in other tissue groups, as well as alveolar macrophages. In lung tissue, IL-4 upregulates the adhesion molecules on the epithelium while regulating the inflammatory response, promotes increased airway responsiveness, and ensures and increases goblet cell hyperplasia; moreover, enhanced immunoglobulin (Ig) E switching, mast cell involvement, and eosinophilia, as well as higher mucus hyperproduction and overall higher airway tissue responsiveness have been described ${ }^{8,17}$. Although we observed a thickening of the basal membranes in some of the patients, generally, no allergic changes in the tissue were found, assuming the production of IL-4 is occurring for other reasons apart from allergic conditions.

Our study shows a more pronounced number of IL-6containing structures in the alveolar epithelium, followed by the number of IL-6-positive structures in the bronchial cartilage and IL-6-containing alveolar macrophages. IL-6 was less found in connective tissue and in bronchial glands, where rare structures and almost none were found, respectively. IL-6 is a pleiotropic cytokine controlling T cell infiltration to the lungs, increasing the mucus secretion, up-regulating the mast cell proliferation, and inducing contractions in lung smooth muscle cells. Importantly, IL-6 mediates the pro-inflammatory, pro-angiogenic and immunomodulatory role of other cells ${ }^{19}$. As observed, IL-6 is produced early during the infection from uninfected cells compared with infected cells, suggesting its regulatory role ${ }^{26}$. As we found, the major sources of IL-6 are structures more exposed to airborne antigens, and the understanding of IL- 6 functions may be explained as a more intermediate occurrence in subsequent signalling with a regulatory role, rather than an initiating factor of immune response induction due to antigen stimulation.

A higher number of IL-7-containing structures were found in bronchial cartilage, followed by location in the bronchial epithelium and the number of IL-7-positive alveolar macrophages. Fewer IL-7-containing structures were found in the alveolar epithelium, connective tissue and bronchial glands. Although IL-7 is found to be secreted by mostly immune cells, as well as supporting cells affecting the development, differentiation and functioning of lymphocytes ${ }^{21}$, our results suggest other non-immune sources. Surprisingly, bronchial cartilage was the most pronounced source for IL-7 affecting the local regulation. However, the role of IL-7 in local immunity is still unclear because the number of studies describing the role of this cytokine in tissues other than lymphoid is limited.

Our study shows that the predominant distribution of IL-10 is higher in bronchial cartilage, ranging from few to numerous IL-10-containing chondrocytes, followed by 
the number of IL-10-containing alveolar macrophages and the number of structures in the bronchial epithelium. Fewer IL-10-containing structures were found in the alveolar epithelium and bronchial glands. Few to a moderate number of interleukin-containing alveolar macrophages, ranging from no positive alveolar macrophages for any other cytokine to numerous IL-10-containing alveolar macrophages were found. However, a lower number of IL-1-containing alveolar macrophages were found. Interestingly, our study results reveal that all structures at first exposed to antigens sourcing from inhaled air (bronchial epithelium, alveolar epithelium, and alveolar macrophages) contained less IL-1 and more IL-10, affecting their own activity in an anti-inflammatory manner. The anti-inflammatory response to the initiated inflammation shows a signalling background between pro-inflammatory and anti-inflammatory cytokines, suggesting that cells capable of expressing IL-10 have powerful sources of controlling the immune response into the anti-inflammatory direction ${ }^{27}$.

As we examined relatively normal tissue without any clinical and microscopic evidence of acute inflammatory condition, our weak findings of IL-1-containing structures confirms the understanding of relatively normal tissue with a preparedness to ignite a full-scale immune response towards inflammation if necessary and dedicated to the status of tissue affected by antigens. Alveolar macrophages unique to lung tissue play a pivotal role in maintaining local immunity. Moreover, alveolar macrophages elicit and further amplify the immune response ${ }^{28,29}$. Importantly, the amount of secreted anti-inflammatory cytokine IL-10 decreases, suggesting the inflammatory response predominance over the tissue anti-inflammatory response, if excited by an antigen. Although epithelial tissue is also a source of cytokines, alveolar macrophages are overall more potent producers of inflammatory mediators than is the epithelium ${ }^{28}$. Antigen exposure causes an increase in acute phase inflammatory cytokines IL-1 $\beta$, IL-6 produced by alveolar macrophages ${ }^{6,7}$.

As an example of cells regulating the immune system, alveolar macrophages are constantly excited by direct exposure to the inhaled antigens through breathing; however, the following pro-inflammatory signalling is strictly controlled and regulated to prevent lung tissue from possible damage due to the full-scale immune response. Not excited by incoming potentially harmful antigens, macrophages function in a relatively silent mode; however, at the same time, alveolar macrophages are ready to be excited. In this state, macrophages suppress the local immunity by producing low levels of cytokines and other signalling molecules ${ }^{29}$. Although alveolar macrophages are found to initiate and maintain the immune response in the inflammatory mode, the contributing factors strongly affect the behaviour of alveolar macrophages. The certain establishment of intercommunication between alveolar macrophages and alveolar epithelial cells directly enhances the immunomodulation in an immunosuppressive way ${ }^{30}$, but we found possible evidence of both paracrine and autocrine regulation by either neighbouring cells or by the cells themselves, respectively.
Although we did not analyse the cytokine contents in the mucus covering the epithelium, we inspected the bronchial glands containing all examined cytokines. Except for few cases where a moderate number of interleukin-containing glandulocytes were found, we obtained an almost negative result, with either no positive cells or mostly rare interleukin-containing glandulocytes. Mucus secreted by both secretory cells and bronchial glands works as a complex defence system containing multiple protective molecules; therefore, we hypothesize that the primary source of cytokines in mucus are not glandulocytes in bronchial glands. Importantly, goblet cells as a one of the secretory cell types are resident and specific to the airway epithelium, where they actively participate in innate and acquired immune responses ongoing throughout the nasal cavity, trachea, conducting bronchi and bronchioles ${ }^{8,9}$. Although induced by IL-13, particularly IL-4, IL-6 and IL-7 are secreted from goblet cells in significantly greater amounts compared with ciliated cells, and these cytokines are expected to also be found in the mucus layer. IL-1RA and IL-10 were also secreted from goblet cells in greater amounts at the apical surface and basolaterally, respectively $^{31}$. Goblet cells apart from other cell types in tissue specimens processed for immunohistochemistry were analysed by semi-quantitative grading, where they showed mostly low-grade colouring. However, this understanding partly fits with our findings in the bronchial epithelium, where all were found up to a mostly moderate number, but in some cases up to numerous interleukin-containing structures.

We found rare to few interleukin-containing structures in the connective tissue, with a greater number of IL10-containing connective tissue fibroblasts. From many pro-inflammatory, modulating and anti-inflammatory cytokines, chemokines and cell adhesion molecules, lung fibroblasts are found to produce IL-1, IL-4, and IL-6. Airway smooth muscle cells have been described to secrete cytokines IL-1, IL-6, and IL-10 (ref. ${ }^{32}$ ). Because of damage to the cellular structure, epithelial cells release IL- $1 \alpha$ to trigger sufficient inflammatory responses in deeper connective tissue fibroblasts ${ }^{33}$. Although epithelial tissue as a lining structure is a more potent producer of cytokines, and the underlying connective tissue shows low, but efficient, amounts of interleukin-containing fibroblasts. A more prominent number of IL-10-containing fibroblasts was found compared with other tissues, suggesting the anti-inflammatory environment was also deeper in the tissue.

Interestingly, we found a prominent source of IL-7 and IL-10 in the bronchial cartilage, where moderate to numerous hypertrophic chondrocytes, as well as chondroblasts positive for IL-7 and IL-10 were found. Although as an early damage pathogen recognition site is in epithelial tissue and alveolar macrophages, other non-immune structures greatly contribute to the initiation, regulation and suppression of the immune response. The epithelium stimulates immune cells and ignites the innate and adaptive immunity signaling 3 ; moreover, alveolar macrophages easily initiate both local and systemic immune responses, as well as recruit other immune mediators, 
leading to inflammation ${ }^{28,29}$. However, the influence of deeper structures to initiate, regulate and suppress the immune responses is poorly evidenced. We suggest the influence of deeper tissue structures in the wall on a larger histological structure, but they mostly regulate the local immune responses by localizing pathological conditions (e.g., inflammation, infection, malignancies, etc.) to prevent spreading throughout the tissue.

We evaluated the relationship between the age of an individual and the number of interleukin-containing structures in all examined tissue, where no statistically significant $(P<0.05)$ correlations were found. Although cellular defects play an important role in the outcomes due to the aging, both local and systemic environments are reorganized towards the inflammation ${ }^{34}$. A persistent low-grade, chronic, systemic pro-inflammatory state described in aging is termed as "inflammaging" or immunosenescence ${ }^{35-37}$. A significant increase in IL-6 and IL-1 $\beta$ due to increasing age was observed. IL-10 was equivalent to the measurements in younger study subjects. An overall basal increase of pro-inflammatory cytokines is observed with aging ${ }^{38}$. Although we evaluated structures containing proinflammatory cytokines, we observed more IL-10-containing structures, suggesting the predominance of antiinflammation is explained as controlling conditions to suppress and regulate the inflammatory environment in relatively healthy lungs regardless of age.

\section{CONCLUSION}

The predominance of IL-10-positive structures in all examined tissue groups indicates the anti-inflammatory response in relatively healthy lung tissue. The weak findings of IL-4-containing structures except in structures more exposed to the outer environment may prove the deficiency of allergic changes in tissue samples mirroring the ontogenetic aspects. The widespread findings of IL-6-positive structures suggest the continuous stimulation of cytokine expression in lung tissue. The relative distribution of IL-7 in all tissue groups indicates the local regulatory and modulating patterns involving non-immune cells and alveolar macrophages. The pronounced distribution of all the cytokines in bronchial cartilage suggests the involvement of a compensatory local immune response in supporting tissues.

Author contributions: This work was conducted as a collaboration between all the authors (ZV: literature search, routine and immunohistochemistry analysis, writing the manuscript, statistical analysis; MP: design of the study, writing the protocol, patient selection, routine and immunohistochemistry analysis; ZV, MP: data interpretation). All authors read and approved the final manuscript.

Conflict of interest statement: The authors state that there are no conflicts of interest regarding the publication of this article.

\section{REFERENCES}

1. McAleer JP, Kolls JK. Directing traffic: IL-17 and IL-22 coordinate pulmonary immune defense. Immunol Rev 2014;260:129-44.

2. Mizgerd JP. Respiratory Infection and the Impact of Pulmonary Immunity on Lung Health and Disease. Am J Respir Crit Care Med 2012;186:824-9.

3. Hallstrand TS, Hackett TL, Altemeier WA, Matute-Bello G, Hansbro PM, Knight DA. Airway epithelial regulation of pulmonary immune homeostasis and inflammation. Clin Immunol 2014;151:1-15.

4. Lambrecht $\mathrm{BN}$, Hammad $\mathrm{H}$. Allergens and the airway epithelium response: Gateway to allergic sensitization. J Allergy Clin Immunol 2014;134:499-507.

5. Vareille M, Kieninger E, Edwards MR, Regamey N. The Airway Epithelium: Soldier in the Fight against Respiratory Viruses. Clin Microbiol Rev 2011;24:210.

6. Chen K, Kolls JK. T Cell-Mediated Host Immune Defenses in the Lung. In: Littman DR, Yokoyama WM, eds. Annu Rev Immunol Vol 31. Palo Alto: Annual Reviews; 2013:605-33.

7. Iwasaki A, Medzhitov R. Control of adaptive immunity by the innate immune system. Nat Immunol 2015;16:343-53.

8. Munkholm M, Mortensen J. Mucociliary clearance: pathophysiological aspects. Clin Physiol Funct Imaging 2014;34:171-7.

9. Evans CM, Koo JS. Airway mucus: The good, the bad, the sticky. Pharmacol Ther 2009;121:332-48.

10. Fahy JV, Dickey BF. Medical Progress: Airway Mucus Function and Dysfunction. N Engl J Med 2010;363:2233-47.

11. Morales-Nebreda L, Misharin AV, Perlman H, Budinger GRS. The heterogeneity of lung macrophages in the susceptibility to disease. Eur Respir Rev 2015;24:505-9.

12. Hashimoto $D$, Chow $A$, Noizat $C$, Teo $P$, Beasley MB, Leboeuf $M$, Becker CD, See P, Price J, Lucas D, Greter M, Mortha A, Boyer SW, Forsberg EC, Tanaka M, van Rooijen N, García-Sastre A, Stanley ER, Ginhoux F, Frenette PS, Merad M. T Tissue-resident macrophages self-maintain locally throughout adult life with minimal contribution from circulating monocytes. Immunity 2013;38:792-804.

13. Lavin Y, Winter D, Blecher-Gonen R, David E, Keren-Shaul H, Merad M, Jung S, Amit I. Tissue-Resident Macrophage Enhancer Landscapes Are Shaped by the Local Microenvironment. Cell 2014;159:1312-26.

14. Garlanda C, Dinarello CA, Mantovani A. The Interleukin-1 Family: Back to the Future. Immunity 2013;39:1003-8.

15. Ranasinghe C, Trivedi S, Wijesundara DK, Jackson RJ. IL-4 and IL13 receptors: Roles in immunity and powerful vaccine adjuvants. Cytokine Growth Factor Rev 2014;25:437-42.

16. Luzina IG, Todd NW, Sundararajan S, Atamas SP. The cytokines of pulmonary fibrosis: Much learned, much more to learn. Cytokine 2015;74:88-100.

17. Lee N, Shin MS, Kang I. T-Cell Biology in Aging, With a Focus on Lung Disease. J Gerontol A Biol Sci Med Sci 2012;67:254-63.

18. Scheller J, Chalaris A, Schmidt-Arras D, Rose-John S. The pro- and anti-inflammatory properties of the cytokine interleukin-6. Biochim Biophys Acta 2011;1813:878-88.

19. Xia YC, Redhu NS, Moir LM, Koziol-White C, Ammit AJ, Al-Alwan L, Camoretti-Mercado B, Clifford RL. Pro-inflammatory and immunomodulatory functions of airway smooth muscle: Emerging concepts. Pulm Pharmacol Ther 2013;26:64-74.

20. Kang J, Coles M. IL-7: The global builder of the innate lymphoid network and beyond, one niche at a time. Semin Immunol 2012;24:1907.

21. Zaunders JJ, Levy Y, Seddiki N. Exploiting differential expression of the IL-7 receptor on memory T cells to modulate immune responses. Cytokine Growth Factor Rev 2014;25:391-401.

22. Guesdon JL, Ternynck T, Avrameas S. The Use Of Avidin Biotin Interaction In Immuno Enzymatic Techniques. J Histochem Cytochem 1979;27:1131-9.

23. Tobin G, Luts A, Sundler F, Ekstrom J. Peptidergic Innervation Of The Major Salivary Glands Of The Ferret. Peptides (N.Y.) 1990;11:863-8.

24. Pilmane M, Rumba I, Sundler F, Luts A. Patterns of occurrence and distribution of neuroendrocine elements in lungs of humans with chronic lung diseases. Proceedings of the Latvian Academy of Sciences Section B Natural Exact and Applied Sciences 1998;52:14452. 
25. Mukaka MM. Statistics Corner: A guide to appropriate use of Correlation coefficient in medical research. Malawi Med J 2012;24:69-71.

26. Copenhaver AM, Casson CN, Nguyen HT, Duda MM, Shin S. IL-1R signaling enables bystander cells to overcome bacterial blockade of host protein synthesis. Proc Natl Acad Sci U S A 2015;112:7557-62.

27. Jin J-O, Han X, Yu Q. Interleukin-6 induces the generation of IL-10producing $\operatorname{Tr} 1$ cells and suppresses autoimmune tissue inflammation. J Autoimmun 2013;40:28-44.

28. Hiraiwa K, van Eeden SF. Contribution of lung macrophages to the inflammatory responses induced by exposure to air pollutants. Mediators Inflamm 2013;2013:619523-619523.

29. Divangahi M, King IL, Pernet E. Alveolar macrophages and type I IFN in airway homeostasis and immunity. Trends Immunol 2015;36:307-14.

30. Westphalen K, Gusarova GA, Islam MN, Subramanian M, Cohen TS, Prince AS, Bhattacharya J. Sessile alveolar macrophages communicate with alveolar epithelium to modulate immunity. Nature 2014;506(7489):503-6. doi: 10.1038/nature12902

31. Tanabe T, Rubin BK. Airway Goblet Cells Secrete Pro-Inflammatory Cytokines, Chemokines, and Growth Factors. Chest 2016;149:714-20.

32. Alkhouri H, Poppinga WJ, Tania NP, Ammit A, Schuliga M. Regulation of pulmonary inflammation by mesenchymal cells. Pulm Pharmacol Ther 2014;29:156-65.

33. Suwara MI, Green NJ, Borthwick LA, Mann J, Mayer-Barber KD, Barron L, Corris PA, Farrow SN, Wynn TA, Fisher AJ, Mann DA IL-1 alpha released from damaged epithelial cells is sufficient and essential to trigger inflammatory responses in human lung fibroblasts. Mucosal Immunol 2014;7:684-93.

34. Kirkwood TBL. Understanding the odd science of aging. Cell 2005;120:437-47.

35. Franceschi C. Inflammaging as a major characteristic of old people: Can it be prevented or cured? Nutr Rev 2007;65:S173-S176.

36. Lalley PM. The aging respiratory system-Pulmonary structure, function and neural control. Respir Physiol Neurobiol 2013;187:199-210.

37. Canan CH, Gokhale NS, Carruthers B, Lafuse WP, Schlesinger LS, Torrelles JB, Turner J. Characterization of lung inflammation and its impact on macrophage function in aging. J Leukoc Biol 2014;96:473480.

38. Moliva JI, Rajaram MVS, Sidiki S, Sasindran SJ, Guirado E, Pan XJ, Wang SH, Ross P Jr, Lafuse WP, Schlesinger LS, Turner J, Torrelles JB. Molecular composition of the alveolar lining fluid in the aging lung. Age (Dordr) 2014;36(3):9633. doi: 10.1007/s11357-014-9633-4 